\title{
FREE SUMMANDS \\ IN MAXIMAL COHEN-MACAULAY APPROXIMATIONS AND EISENBUD OPERATORS OVER HYPERSURFACE RINGS
}

\author{
ALEX MARTSINKOVSKY
}

(Communicated by Maurice Auslander)

\begin{abstract}
The Eisenbud operator of a module over a complete hypersurface ring completely determines the delta invariants of this module.
\end{abstract}

\section{INTRODUCTION}

The main goal of this paper is to investigate the absence (or presence) of free summands in maximal Cohen-Macaulay (MCM) approximations over hypersurface rings $R=S /(f)$, where $S$ is a formal power series ring over a field. The importance of this problem first manifested itself in the form of a criterion for quasihomogeneity of $R$ (in the case when $R$ is an isolated singularity): $R$ is quasihomogeneous if and only if the MCM approximation of the moduli algebra $R / \overline{j(f)}$ of $R$ has no free summands (see [Mar 1, Mar 2]).

Recently Ding undertook a first systematic study [Ding] of this phenomenon over Gorenstein rings. Among other things, he exhibited interesting connections with multiplicities and deformation theory. Working over the ring $R=S /(x)$, where $\left(S, \underline{m}_{s}\right)$ is a complete local Gorenstein ring and $x \in \underline{m}_{s}$ is an $S$ regular element, he also gave, for a finitely generated $R$-module $N$, a sufficient condition for the absence of free summands in its MCM approximation. More precisely, let $\delta(N)$ (respectively, $\delta^{i}(N)$ ) denote the number of copies of $R$ in the minimal MCM approximation of $N$ (respectively, the $i$ th syzygy module $\Omega^{i} N$ of $\left.N\right)$. He then proves

Theorem 1.1 [Ding, Theorem 2.5]. If $x \in \underline{m}_{s} \operatorname{Ann}_{s} N$ then $\delta^{i}(N)=0$ for all $i \geq 0\left(\right.$ here $\left.\delta^{0}(N):=\delta(N)\right)$.

Moreover, if $R$ is a hypersurface ring with $x \in \underline{m}_{s}^{2}$ and $N$ is cyclic, the converse is true.

Theorem 1.2 [Ding, Theorem 2.11]. If $N=R / \bar{a}$ then $\delta(R / \bar{a})=0$ if and only if $x \in \underline{m}_{s} a$, where $a$ is the preimage of $\bar{a}$ in $S\left(=\mathrm{Ann}_{s} R / \bar{a}\right)$. If $\delta(R / \bar{a})=0$ then $\delta^{i}(R / \bar{a})=0$ for all $i \geq 0$.

Received by the editors August 23, 1990 and, in revised form, January 14, 1991.

1991 Mathematics Subject Classification. Primary 13C99.

Key words and phrases. Maximal Cohen-Macaulay approximation, Eisenbud operator, delta invariant. 
Theorem 1.1 is a powerful result; however, in the noncyclic case its converse is not true. Thus we would like to know criteria or at least necessary conditions for the equalities $\delta(N)=0$ and $\delta^{i}(N)=0 \quad \forall i \geq 0$.

In this paper we obtain a criterion for the equalities $\delta^{i}(N)=0 \forall i \geq 0$ and a necessary condition for the equality $\delta(N)=0$. They turn out to be completely elementary and are formulated in terms of the Eisenbud operator of $N$, i.e., a degree -2 map $t: \mathbb{F} \rightarrow \mathbb{F}$, where $\mathbb{F}$ is a minimal projective resolutic.. of the $R$-module $N$

$$
\cdots \stackrel{d_{n}}{\longrightarrow} F_{n} \stackrel{d_{n-1}}{\longrightarrow} \cdots \longrightarrow F_{2} \stackrel{d_{1}}{\longrightarrow} F_{1} \stackrel{d_{0}}{\longrightarrow} F_{0} \longrightarrow N \longrightarrow 0 .
$$

More precisely, if $\delta(N)=0$ then $t_{0}: F_{2} \rightarrow F_{0}$ is necessarily an epimorphism. Almost immediately, this leads us to the following criterion: $\delta^{i}(N)=0$ for all $i \geq 0$ if and only if the Eisenbud operator $t: \mathbb{F} \rightarrow \mathbb{F}$ is an epimorphism (by [Eis] it suffices to check that $t_{i}: F_{i+2} \rightarrow F_{i}$ is an epimorphism for $\left.i \leq \operatorname{dim} R+1\right)$. We should remark that once a truncation

$$
\mathbb{F}^{n+2} \quad F_{n+2} \longrightarrow F_{n+1} \longrightarrow F_{n} \longrightarrow \cdots \longrightarrow F_{0} \longrightarrow N \longrightarrow 0
$$

of $\mathbb{F}$ is known, the verification of the condition " $t_{i}: F_{i+2} \rightarrow F_{i}$ is an epimorphism for $i \leq n$ " is trivial (it reduces to computing the rank of a matrix with entries in a field).

If $N$ happens to be a cyclic module then our necessary condition becomes a criterion: $\delta(R / \bar{a})$ if and only if $t_{0}: F_{2} \rightarrow F_{0}$ is an epimorphism. It is interesting to observe that while Ding's sufficient condition becomes necessary in the cyclic case, our necessary condition becomes sufficient. Thus for cyclic modules the two conditions become equivalent (this can also be verified directly). In the cyclic case our condition can, with little work, be deduced from Ding's theorems. However, we provide a different proof hoping that it may also work, with necessary modifications, for noncyclic modules. This would make the condition " $t_{0}: F_{2} \rightarrow F_{0}$ is an epimorphism" into a criterion for $\delta(N)=0$. In fact, the problem boils down to finding a suitable generalization of the Koszul complex, including its multiplicative structure.

In any event we feel that the Eisenbud operator should be carefully studied if we want to understand the behaviour of the numbers $\delta^{i}(N)$. It suffices to remark that rkCoker $t_{i} \leq \delta^{i}(N)$ for any $i$ (with the possibility that the two numbers are actually equal not ruled out yet!).

\section{BACKGROUND MATERIAL}

2.1. The Eisenbud operator (see [Eis] for details). Let $(A, \underline{m})$ be a regular local ring, $x \in \underline{m}$, and $B:=A /(x)$. Also let

$$
\cdots \longrightarrow F_{i+2} \stackrel{\partial}{\longrightarrow} F_{i+1} \stackrel{\partial}{\longrightarrow} F_{i} \longrightarrow \cdots
$$

be a complex of free $B$-modules. Lifting $F_{i}$ 's and $\partial$ to $A$ we obtain a sequence of free modules and maps between them 
Since $\partial^{2}=0$, we have that $\tilde{\partial}^{2}$ can be written (nonuniquely) as $x \tilde{t}$, where $\tilde{t}$ is a map $\widetilde{F}_{i+2} \rightarrow \widetilde{F}_{i}$ (for each $i$ ). We now set $t:=\tilde{t} \otimes B$; this is a degree -2 map $\mathbb{F} \rightarrow \mathbb{F}$. It is clear that $t$ is not unique (since liftings are not unique). However, $t$ turns out to be a homomorphism of complexes of degree -2 , and as such it is determined uniquely up to homotopy. Its homotopy class will be called the Eisenbud operator of $\mathbb{F}$ and denoted, without the danger of confusion, by the same letter $t$. In particular, for any $B$-module $M$ the induced maps $t_{i}: H_{i+2}(\mathbb{F} \otimes M) \rightarrow H_{i}(\mathbb{F} \otimes M)$ and $t^{i}: H^{i}(\operatorname{Hom}(\mathbb{F}, M)) \rightarrow H^{i+2}(\operatorname{Hom}(\mathbb{F}, M))$ are determined uniquely. If $\mathbb{F}$ is a $B$-free resolution of a $B$-module $N$, then we obtain natural transformations $t_{i}: \operatorname{Tor}_{i+2}^{B}(N,-) \rightarrow \operatorname{Tor}_{i}^{B}(N,-)$ that do not depend on the choice of $\mathbb{F}$. If $\mathbb{F}$ is a minimal free resolution of $N$ then $t$ will be called the Eisenbud operator of $N$. In this case, it makes sense to speak of surjectivity of $t$. Indeed the homotopy $h$ between the two representatives of the Eisenbud operator corresponding to liftings $\tilde{\partial}$ and $\tilde{\partial}^{\prime}$ is given by the formulas $\tilde{\partial}-\tilde{\partial}^{\prime}=x \tilde{h}$ and $h:=\tilde{h} \otimes B$ [ibid, Proposition 1.3]. Hence $h$ is a homomorphism of modules. Since $\partial$ has entries in the maximal ideal, a representative is surjective if and only if any other representative is. It is obvious that, under the above assumptions, $t_{i}: F_{i+2} \rightarrow F_{i}$ is surjective if and only if $t_{i}: \operatorname{Tor}_{i+2}^{B}(N, k) \rightarrow \operatorname{Tor}_{i}^{B}(N, k)$ is surjective.

2.2. Matrix factorizations (see [Eis] for details). Let $(A, \underline{m})$ be a regular local ring and $x \in m$. A matrix factorization of $x$ is an ordered pair $(\varphi: F \rightarrow G$, $\psi: G \rightarrow F$ ) of homomorphisms of free $A$-modules $F$ and $G$ such that $\varphi \psi=$ $x \cdot \operatorname{Id}_{G}$ and $\psi \varphi=x \cdot \operatorname{Id}_{F}$. In any such pair, $F$ and $G$ have the same rank. A factorization $(\varphi, \psi)$ is called reduced if $\operatorname{Im} \varphi \subset \underline{m} G$ and $\operatorname{Im} \psi \subset \underline{m} F$.

Now consider the ring $B:=A /(x)$. If $(\varphi, \psi)$ is a matrix factorization of $x$ then the $A$-module Coker $\varphi$ (and Coker $\psi$ ) is annihilated by $x$ and thus becomes a $B$-module. It turns out that this module is MCM. Moreoever, any MCM over $B$ can be obtained this way. The $B$-module Coker $\varphi$ has no free summands if and only if $(\varphi, \psi)$ is reduced, and if this is the case then

$$
\cdots \longrightarrow \bar{F} \stackrel{\bar{\varphi}}{\longrightarrow} \bar{G} \stackrel{\bar{\psi}}{\longrightarrow} \bar{F} \stackrel{\bar{\varphi}}{\longrightarrow} G
$$

is a minimal $B$-free resolution of Coker $\varphi$ (overbar denotes reduction modulo $(x))$.

2.3. MCM approximations over hypersurface rings (see [Aus B, Ding] for details). Let $\left(S, \underline{m}_{S}\right)$ be a formal power series ring over a field, $x \in \underline{m}_{S}^{2}, R:=$ $S /(x)$, and $C$ an $R$-module. An exact sequence $0 \rightarrow Y_{C} \rightarrow X_{C} \stackrel{p}{\rightarrow} C \rightarrow 0$ of $R$-modules is an MCM approximation of $C$ if $X_{C}$ is MCM and $Y_{C}$ is of finite injective dimension. Since $R$ is Gorenstein, the latter is equivalent to saying that $Y_{C}$ is of finite projective dimension. This approximation is called minimal if the map $p$ is right minimal, i.e., no direct summand of $X_{C}$ is mapped to 0 by $p$. Approximations always exists and the minimal ones are determined uniquely up to (noncanonical) isomorphisms. Henceforth we will only deal with minimal approximations.

MCM approximations over the hypersurface ring $R$ can be constructed as follows. Let $n:=\operatorname{dim} R$. Then by [Eis], a minimal resolution of $C$ becomes 
(at most) 2-periodic no later than at the $(n+1)$ st step:

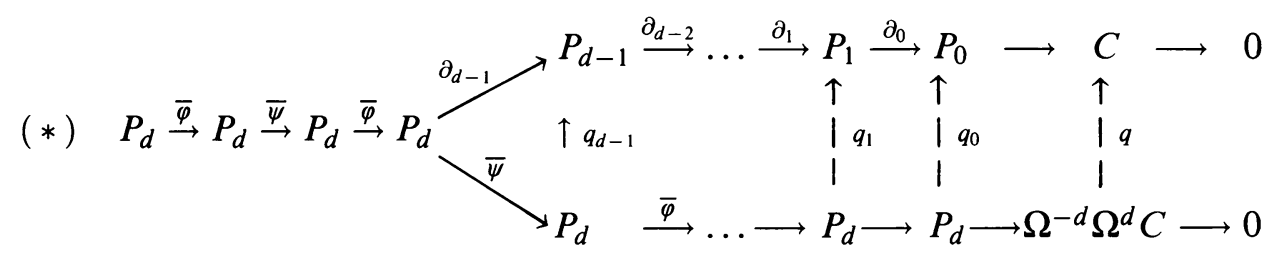

Here $d \leq n$ and $(\varphi, \psi)$ is a matrix factorization of $x$. Pushing out the identity map on $\Omega^{d} C \cong \Omega^{d} \Omega^{-d} \Omega^{d} C$ ( $\Omega^{d}$ stands for the $d$ th syzygy module), we obtain vertical maps $q_{i}: P_{d} \rightarrow P_{i}$ and $q: \Omega^{-d} \Omega^{d} C \rightarrow C$. Now let $q^{\prime}: F \rightarrow C / \operatorname{Im} q$ be a projective cover. Then, upon identifying $q^{\prime}$ with its lifting to $C$, we obtain the desired minimal MCM approximation: $q \amalg q^{\prime}: \Omega^{-d} \Omega^{d} C \amalg F \rightarrow C$ (of course, if $q$ is surjective then it is already a minimal approximation). Now it follows immediately that $\delta(C)=\operatorname{rk} F$ and $\delta^{i}(C)=0$ if and only if the map $q_{i}$ is onto, where $i$ is any nonnegative integer.

\section{The NUMBers $\delta^{i}$ AND THE EISENBUd OPERATOR}

Throughout the rest of this paper we keep the notation from $\S 2.3$. For ease of reference we introduce the following

Definition 3.1. (a) An $R$-module $N$ will be called a module of type $A$ if the degree zero part $t_{0}: F_{2} \rightarrow F_{0}$ of the Eisenbud operator of $N$ is surjective;

(b) an $R$-module $N$ will be called a module of type $B$ if the Eisenbud operator $t: \mathbb{F} \rightarrow \mathbb{F}$ of $N$ is surjective.

Lemma 3.2. Let $\alpha: M \rightarrow N$ be a surjective homomorphism of $R$-modules, and assume that $M$ is of type $A$. Then $N$ is also of type $A$.

Proof. The assertion of the lemma follows form the naturality of the Eisenbud operator (see [Eis, Proposition 1.3]). More precisely, let $s$ (respectively, $t$ ) be the Eisenbud operator of $m$ (respectively, $N$ ). Then [ibid] $\alpha_{*} s$ and $t \alpha_{*}$ give rise to the same homotopy class of maps. But $\alpha_{0} s_{0}$ is onto. Therefore $t_{0} \alpha_{2}$ is onto and we have the desired result.

Corollary 3.3. If, under the assumptions of the previous lemma, $M$ is of type $B$ and the chain map $\alpha_{*}$ is surjective (i.e., each homogeneous part $\alpha_{i}$ of $\alpha_{*}$ is surjective), then $N$ is also a type $B$ module.

Now we can give a necessary condition for the equality $\delta(N)=0$.

Proposition 3.4. Let $N$ be an $R$-module with $\delta(N)=0$. Then $N$ is of type $A$. Proof. Since $\delta(N)=0$, there exists an MCM module $M$ without free summands (e.g., the MCM approximation of $N$ ) mapping onto $N$. But $M$ comes from a reduced matrix factorization, say $(\Delta, \nabla)$, so that $M \cong_{R}$ Coker $\bar{\Delta}$ and $\Delta \nabla=x \cdot$ Id where Id is the identity map. Thus the identity map can be chosen as a representative of the Eisenbud operator of $M$. Therefore $M$ is a type $A$ module, and the desired result follows from Lemma 3.2.

In the same vein we shall now give a necessary and sufficient condition for the equalities $\delta^{i}(N)=0, i \geq 0$. 
Proposition 3.5. Let $N$ be an R-module. Then $\delta^{i}(N)=0$ for all $i$ if and only if $N$ is a type B-module.

Proof. The "only if" part is obvious (just apply Proposition 3.4 to $\Omega^{i} N$ ). To prove the "if" part, consider commutative diagram $(*)$ of $\S 2.3$. We want to show that $q_{i}$ is onto for all $i$. We already know that $q_{i}$ is onto for $i \geq d$ (those $q_{i}$ are just identity maps). We proceed by the inverse induction on $i$. It suffices to show that $q_{d-1}$ is onto. Consider the commutative diagram

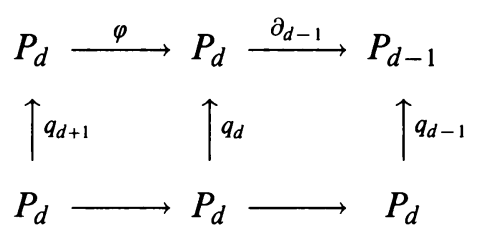

Let $t$ (respectively, $s$ ) be the Eisenbud operator of $N$ (respectively, $\Omega^{-d} \Omega^{d} N$ ). Again, by the naturality of the Eisenbud operator, $t q_{*}$ and $q_{*} s$ give rise to the same homotopy class of maps, but $t_{d-1} q_{d+1}$ is onto by hypothesis. Therefore $q_{d-1} s_{d-1}$ is also onto, and thus $q_{d-1}$ is an epimorphism.

Remarks. (1) Once a minimal projective resolution of $N$ is known, the condition " $t_{i}$ is onto" is very easy to verify: just lift the differential to $S$, compute the square of the lifting (and we only need the initial form of that), and divide the result by $x$ (and we only need the constant term of the result!). After that, of course, it remains to determine the rank of a matrix with entries in a field.

The following remark was suggested by the referee:

(2) Via induction it is not difficult to show that the maps $q_{i}$ can be chosen in the following form:

$$
q_{d-1}= \begin{cases}t_{d-i} t_{d-i+2} \cdots t_{d-1}, & \text { when } i \text { is odd, } \\ t_{d-i} t_{d-i+2} \cdots t_{d-2}, & \text { when } i \text { is even. }\end{cases}
$$

Thus the question of surjectivity of the maps $q_{i}$ can be completely answered if a minimal projection resolution of $N$ is known.

(3) If $N$ is an $R$-module of type $A$ then, since $t_{0}$ is onto, the lifting $S^{n_{2}} \stackrel{\tilde{\partial}_{1}}{\rightarrow}$ $S^{n_{1}} \stackrel{\partial_{0}}{\rightarrow} S^{n_{0}}$ of $\partial_{0} \partial_{1}$ to $S$ can be written as $x \cdot(U \mid V)$, where $x$ is the $n_{0} \times n_{0}$ scalar matrix with $x$ on the diagonal, and $(U \mid V)$ is a block matrix with $U$ an invertible $n_{0} \times n_{0}$ matrix and $V$ an $n_{0} \times\left(n_{2}-n_{0}\right)$ matrix. By changing basis, if necessary, we can make $U$ the identity matrix and $V$ the zero matrix, i.e., $\tilde{\partial}_{0} \tilde{\partial}_{1}=x \cdot(\operatorname{Id} \mid 0)$. In particular, if $N$ is a cyclic module $R / a$ then $t_{0}$ is surjective if and only if $x \in \underline{m}_{s} a^{c}=\underline{m}_{s} \mathrm{Ann}_{s} R / a$. Notice also that rk $\tilde{\partial}_{0} \tilde{\partial}_{1}=n_{0}$. These simple observations give us the following weaker, yet useful, results.

Corollary 3.6. Let $R^{n_{1}} \rightarrow R^{n_{0}} \rightarrow N \rightarrow 0$ be a (not necessarily minimal) presentation of an R-module $N$. If $n_{1}<n_{0}$ then $\delta(N)>0$.

Corollary 3.7. Let $S^{n_{2}} \stackrel{\tilde{\partial}_{1}}{\rightarrow} S_{1}^{n} \stackrel{\tilde{\partial}_{0}}{\rightarrow} S^{n_{0}}$ be a lifting of the beginning of a minimal projective resolution of an $R$-module $N, m$ the degree of $x$ (i.e., the degree of the initial form of $x$ ), and $m_{0}$ (respectively, $m_{1}$ ) the minimum of the degrees of the entries of $\tilde{\partial}_{0}$ (respectively, $\left.\tilde{\partial}_{1}\right)$. If $m<m_{0}+m_{1}$ then $\delta(N)>0$. 
The last result can be colloquially paraphrased as "high multiplicity implies positivity of $\delta$."

\section{THE CYCLIC CASE}

Proposition 4.1. Let $N \cong R / a$ be a cyclic $R$-module. Then $\delta(N)=0$ if and only if $N$ is of type $A$.

Remark. As follows from Remark (3) in $\S 3$, the desired assertion can be easily deduced from Ding's Theorem 2 (see Introduction). However, we give a different proof hoping that it could be generalized to the noncyclic case. (Ding's Theorem 1, which was used in the proof of Theorem 2, is not true in the noncyclic case).

Proof of Proposition 4.1. The "only if" part was proved in Proposition 3.4. To prove the "if" part we will explicitly construct an MCM $R$-module without free summands and a surjective map from that module to $N$.

Suppose that the contraction $a^{c}$ of $a$ to $S$ is generated by elements $a_{1}, \ldots$, $a_{t} \in S$. As was mentioned before, we have that $x \in \underline{m}_{s} a^{c}$, i.e., $x=\sum_{i=1}^{t} y_{i} a_{i}$, where $y_{i} \in \underline{m}_{s}, i=1, \ldots, t$. To find a suitable MCM module we utilize matrix factorization $(\delta, \delta)$ from [BGS]. For the sake of completeness we recall its construction. Let $S^{t}$ be the free $S$-module on generators $e_{1}, \ldots, e_{t}$. The map $S^{t} \stackrel{\left(a_{1} \ldots a_{t}\right)}{\rightarrow} S$ gives rise to the Koszul complex $\wedge^{\bullet} S^{t}$ with differential $\delta_{-1}:=$ $\sum_{i=1}^{t} a_{i} e_{i}^{*}: \Lambda^{\bullet} S^{t} \rightarrow \Lambda^{\bullet} S^{t}$, where $\left\{e_{i}^{*}\right\}_{i=1}^{t}$ is the basis of $\left(S^{t}\right)^{*}$ dual to $\left\{e_{i}\right\}_{i=1}^{t}$ and denotes the contraction operator. Also define $\delta_{+1}: \Lambda^{\bullet} S^{t} \rightarrow \Lambda^{\bullet} S^{t}$ as the exterior multiplication by $\sum_{i=1}^{t} y_{i} e_{i}$, and let $\delta:=\delta_{+1}+\delta_{-1}: \bigwedge^{\bullet} S^{t} \rightarrow$ $\Lambda^{\bullet} S^{t}$. As was shown in [ibid], $(\delta, \delta)$ is a reduced matrix factorization of $x=\sum y_{i} a_{i}$. (Proof: multiplication by $x=\sum y_{i} a_{i}$ is null-homotopic; the homotopy is given by $\delta_{+1}$.) Thus we have an MCM $R$-module without free summands with $\Lambda^{\bullet} R^{t} \stackrel{\bar{\delta}}{\rightarrow} \Lambda^{\bullet} R^{t} \rightarrow M$ as minimal presentation. We now define $\alpha_{0}: \Lambda^{\bullet} R^{t} \rightarrow R$ and $\alpha_{1}: \Lambda^{\bullet} R^{t} \rightarrow R^{t}$ as projections onto the summands $\bigwedge^{0} R^{t}$ and, respectively, $\bigwedge^{1} R^{t}$. Then $\alpha_{0} \bar{\delta}=\left(\bar{a}_{1} \ldots \bar{a}_{t}\right) \alpha_{1}$, which yields the desired surjection $\alpha: M \rightarrow R / a$.

Remark. A natural question to ask is whether $\delta(N)=0$ for noncyclic $R$ modules of type $A$. The affirmative answer would then give us a convenient criterion for the vanishing of $\delta$.

\section{REFERENCES}

[AusB] M. Auslander and R.-O. Buchweitz, The homological theory of maximal CohenMacaulay approximations, Mém. Soc. Math. France 38 (1989), 5-37.

[BGS] R.-O. Buchweitz, G.-M. Greuel, and F.-O. Schreyer, Cohen-Macaulay modules on hypersurface singularities. II, Invent. Math. 88 (1987), 165-182.

[Ding] S. Ding, Cohen-Macaulay approximations over a Gorenstein local ring, Thesis, Brandeis Univ., 1990.

[Eis] D. Eisenbud, Homological algebra on a conplete intersection, with an application to group representations, Trans. Amer. Math. Soc. 260 (1980), 35-64. 
[Mar1] A. Martsinkovsky, Almost split sequences and Zariski differentials, Thesis, Brandeis Univ., 1987.

[Mar2] __ Maximal Cohen-Macaulay modules and the quasihomogeneity of isolated CohenMacaulay singularities, Proc. Amer. Math. Soc. 112 (1991), 9-18.

Department of Mathematics, Northeastern University, 360 Huntington Avenue, Boston, MassachusetTs 02115

E-mail address: alexmart@nuhub.bitnet 